Research Paper

\title{
Targeting the mTOR pathway in Chromophobe Kidney Cancer
}

\section{Brian Shuch ${ }^{1}$, Srinivas Vourganti1 ${ }^{1}$ Julia C. Friend1', Lee M. Zehngebot² ${ }^{2}$ W. Marston Linehan¹, Ramaprasad Srinivasan ${ }^{1 \otimes}$}

1. Urologic Oncology Branch, National Cancer Institute, 10 Center Drive, CRC, Building 10, Room 1-5940, Bethesda, MD 20892, USA;

2. Orlando Oncology, Orlando, Florida, 2501 N. Orange Avenue, Ste. 381, Orlando, FL 32804, USA.

$\triangle$ Corresponding author: Ramaprasad Srinivasan, M.D., Ph.D. Senior Clinician, Urologic Oncology Branch, National Cancer Institute, 10 Center Drive, CRC 2-5950, Bethesda, MD20892, Tel: (301) 496-6353, Fax: (301) 402-0922 ramasrin@mail.nih.gov

(C) Ivyspring International Publisher. This is an open-access article distributed under the terms of the Creative Commons License (http://creativecommons.org/ licenses/by-nc-nd/3.0/). Reproduction is permitted for personal, noncommercial use, provided that the article is in whole, unmodified, and properly cited.

Received: 2012.03.17; Accepted: 2012.03.30; Published: 2012.04.02

\begin{abstract}
Chromophobe kidney cancer accounts for approximately $5 \%$ of cases of renal cell carcinoma (RCC). While the genetics of clear cell RCC has been a major focus of research, little is known about the biology of chromophobe tumors. There is ample preclinical rationale for the use of targeted therapy in clear cell tumors, and agents targeting the VHL/HIF pathway are now widely used in clinical practice. However, there is limited experience with targeted agents in non-clear cell tumors. Recently, a few case reports have emerged which report the use of mTOR inhibitors in chromophobe tumors. Here, we report our experience with targeted therapy in a patient with advanced chromophobe RCC who had a durable partial response to temsirolimus. We also include a literature review summarizing the published experience with targeted therapeutic approaches in chromophobe RCC. Additionally, the preclinical rationale for the use of mTOR inhibitors in this population based on our characterization of the hereditary form of chromophobe kidney cancer, Birt-Hogg-Dube syndrome, is discussed.
\end{abstract}

Key words: mTOR inhibitors, Chromophobe Kidney Cancer

\section{Introduction}

Advances in our understanding of the genetic alterations underlying clear cell renal cell carcinoma (RCC) has led to a proliferation of clinically active agents for this disease, and since 2005, a total of seven agents (sorafenib, sunitinib, temsirolimus, bevacizumab, everolimus, pazopanib, and axitinib) have been approved for the treatment of metastatic kidney cancer. While kidney cancer was once considered to be a single entity, extensive research over the past 3 decades has resulted in classification of RCC into distinct subtypes based on disparate genetic and molecular alterations. Most of the FDA approved agents have been tested in a fairly uniform patient population with predominantly clear cell tumors. Extrapolating the benefit of these agents to the other common subtypes of kidney cancer including papillary and chromophobe tumors, while common in clinical practice, is not supported by robust scientific and clinical evidence. Thus, clinical trial participation should be encouraged for patients with non-clear cell histology, a recommendation endorsed by the NCCN. ${ }^{1}$ Several small phase II trials have looked at the use of targeted agents in the patients with papillary and other non-clear cell variants of RCC and have not demonstrated an overwhelming benefit. ${ }^{2,3}$

The clinical utility of inhibitors of the VEGF or mTOR pathways in patients with chromophobe RCC remains unclear with limited evidence acquired from retrospective analyses and expanded access trials. Choueiri and colleagues described their experience 
with VEGF pathway antagonists in a multi-institutional, retrospective series, reporting 3 partial responses in 12 patients. ${ }^{4}$ Data from the expanded access program for sorafenib also suggests some activity in chromophobe RCC as $90 \%$ of patients $(n=18)$ had some measure of disease control ( 1 partial response and 17 stable disease). ${ }^{5}$ For mTOR agents, the results are more uncertain with no large case series or prospective studies available. Post hoc subgroup analysis of the ARCC study with temsirolimus demonstrated a potential benefit in non-clear cell RCC; however the incidence of chromophobe RCC in the non-clear group is not reported. ${ }^{6}$

In order to better understand the potential role of mTOR, we evaluated the literature and our institutional experience with patients with metastatic chromophobe RCC. Additionally we sought to better understand the burden of metastatic chromophobe RCC in the United States using the Surveillance Epidemiology and End Result (SEER)-17 program.

\section{Methods}

We reviewed the literature for published reports of chromophobe RCC treated with mTOR therapy from 2006-2011. Search terms included "chromophobe" and "kidney cancer." All manuscripts were reviewed for patients with metastatic chromophobe RCC who had received systemic 'targeted therapy' including sirolimus, everolimus, or temsirolimus. Reports selected had details outlining the agent used, indication, line of therapy, prior treatment, response, and patient outcome. Our institutional database was reviewed to determine cases of metastatic chromophobe RCC from 2006-2011 treated with mTOR therapy. Only one patient was identified and we reviewed the patient's presentation, surgical management, systemic therapy, and clinical response.

A brief review of the Surveillance Epidemiology and End Result (SEER)-17 program provided an estimate of the deaths due to chromophobe RCC each year. The SEER program now records cancer statistics from approximately $28 \%$ of the United States population. From 2003 to 2008, all patients listed as having cancer of the kidney and renal pelvis were selected. Cases with ICD-O-3 histology codes believed to represent RCC (8140, 8260, 8270, 8290, 8310, 8312, 8316, 8317-8320) were chosen for further review. Patients dying of RCC from 2003-2007 were reviewed to determine the number of deaths attributed to chromophobe RCC (histology codes 8270 and 8317). The annual death rates per 100,000 was calculated for RCC overall and for chromophobe histology using SEER ${ }^{\star}$ Stat (Version 7.05).

\section{Results}

\section{Case Report}

Review of our institutional database revealed a single patient with metastatic chromophobe RCC treated with targeted therapy. In February, 2000, a 45 year-old woman presented with fatigue, weight loss, and a large palpable abdominal mass. A CT scan of the abdomen demonstrated an $18 \mathrm{~cm}$ left renal mass (Figure 1). She underwent a left sided radical nephrectomy and final pathology demonstrated a T2, chromophobe renal cell carcinoma (RCC) with areas of sarcomatoid transformation. She was followed closely with periodic surveillance and was free of disease for 6 years. In June 2006, she presented with multiple small liver nodules; laparoscopic resection of a hepatic lesion confirmed the presence of metastatic chromophobe RCC. Once recovered, she was placed on sunitinib (50mg orally on a four-weeks on, two-weeks off schedule) and had regression in some of the liver lesions.

Following five cycles (approximately 7 months), she demonstrated progressive disease, with enlargement of several liver lesions (largest approximately $2.5 \mathrm{~cm}$ ). Sunitinib was discontinued after June of 2007 and temsirolimus was recommended $(25 \mathrm{mg} / \mathrm{kg}$ IV every week). The patient was unable to promptly initiate therapy and started temsirolimus around the time of repeat imaging in October 2007 at which time the largest liver lesion had increased to $3.2 \mathrm{~cm}$. On therapy, multiple liver lesions regressed, and the patient achieved a partial response per RECIST. In February 2008, a chest CT obtained for restaging revealed mild interstitial changes. However, the patient had no accompanying symptoms and pulmonary function testing was normal; temsirolimus was therefore continued with close monitoring for respiratory symptoms.

During the next two years the liver lesions remained stable on therapy. However, in August, 2009, she developed several small bilateral sub-centimeter pulmonary nodules, consistent with lung metastases. Bevacizumab was added to temsirolimus at that time. In June, 2010 she developed significant proteinuria and bevacizumab was discontinued. Fortunately, the lung and liver lesions have remained fairly stable with minimal interval growth of some lesions. Currently, she remains on temsirolimus with excellent performance status, alive 5 years after the development of metastatic disease. Figure 2 summarizes her treatment history and clinical course. 


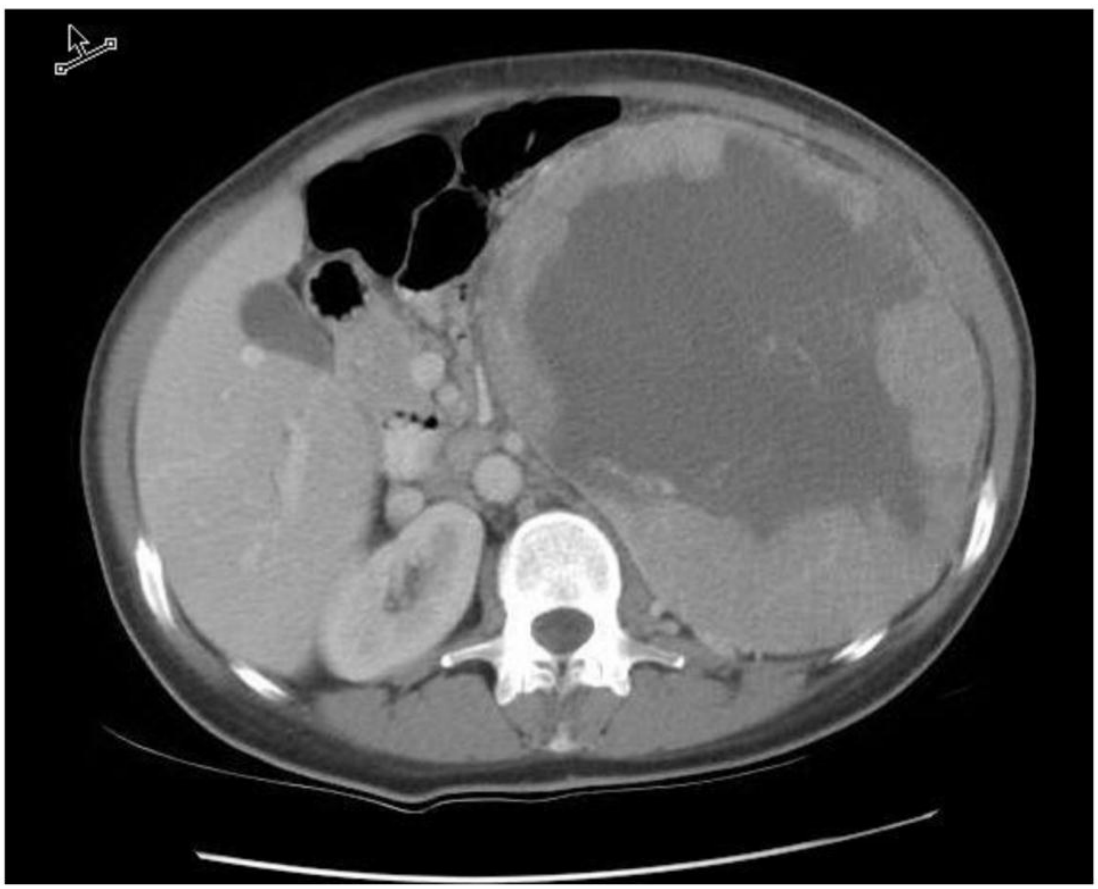

Figure I: CT scan with IV contrast demonstrating the $18 \mathrm{~cm}$ left renal tumor prior to radical nephrectomy in 2002.

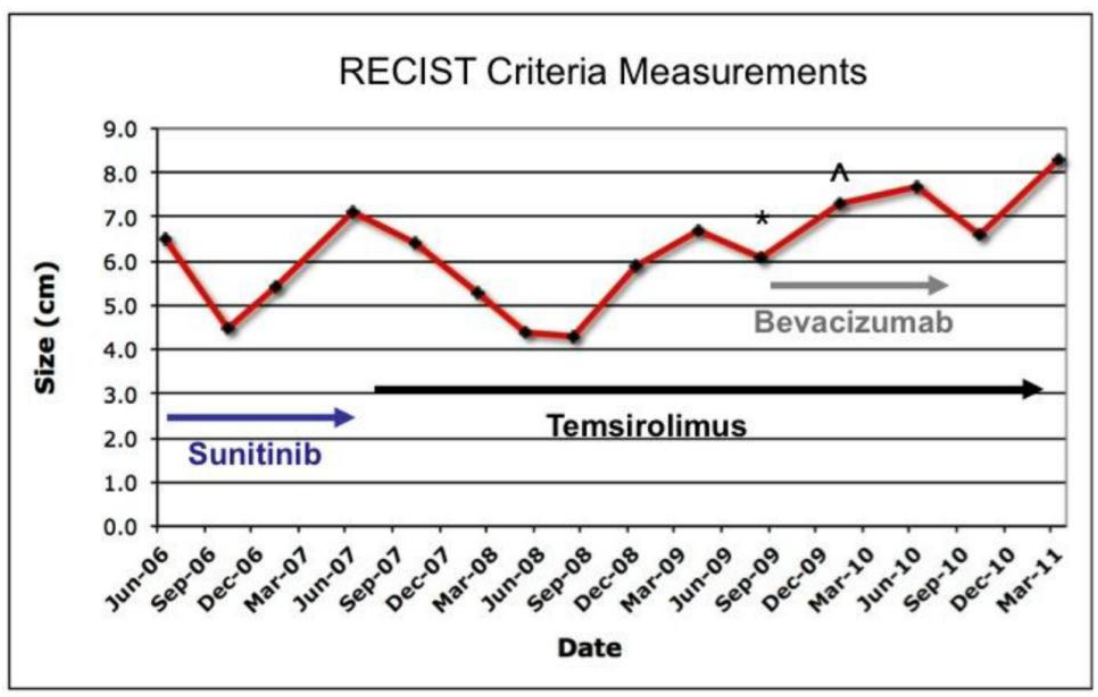

Figure 2: Treatment history and total disease progression calculated by summation of maximal dimension of all measurable lesions $(>1$ $\mathrm{cm}$ ) in axial imaging (RECIST). (*Small lung nodules noted on chest imaging). (^ Appearance of measurable lung nodules).

\section{Published Reports}

In the past year, a small number of interesting case reports have described the activity of mTOR agents in chromophobe RCC (Table 1).7-9 The first report from Paule and colleagues described a 57 year-old man who had an early retroperitoneal recurrence after a left radical nephrectomy for a T2 Fuhrman grade 2-3 chromophobe RCC. ${ }^{8}$ The patient had initial treatment with subcutaneous Interferon-alpha followed by sorafenib but eventually progressed. Temsirolimus therapy lead to disease stabilization for 26 months and the patient had a partial resection of the retroperitoneal mass. The patient resumed therapy and remained on treatment for over two years. The last imaging study showed a significant response of the residual retroperitoneal mass per author assessment. 
Zardavas and colleagues report a case of a 27 year-old female who underwent a radical nephrectomy for a T2N1M0 chromophobe RCC. ${ }^{9}$ The patient presented three years later with metastatic disease in the liver and received sunitinib and then sorafenib therapy without an objective response. The patient was placed on temsirolimus and had a partial response by RECIST criteria and remains on therapy after a year with excellent performance status.

The final report from Larkin and colleagues described a 53 year-old man who underwent a right radical nephrectomy for a $6 \mathrm{~cm}$, T3a, Fuhrman grade III chromophobe RCC.7 The patient was found to have an unresectable retroperitoneal recurrence three years later and was placed on sunitinib. After a partial response the patient was taken off therapy for unrelated medical issues. Everolimus was started and the patient experienced a partial response by RECIST criteria, still remaining on mTOR therapy after 2 years.

\section{SEER Population Estimates}

From 2003-2008, the annual adjusted death rate per 100,000 persons for RCC overall ranged from 2.670 to 3.091 . For chromophobe histology, the death rate was extremely low, ranging from 0.015 to 0.034 per 100,000 persons (Figure 3A). The number of SEER cases of deaths from chromophobe RCC identified was between 4-9 cases/year and accounted for approximately $1 \%$ of total deaths from RCC (Figure 3B). As SEER data relies on medical extraction, the numbers may have some inaccuracies. However, based on this data, the number of total chromophobe deaths a year in the US population would be roughly 14-32 per year.

\section{Discussion}

Unlike clear cell RCC, the natural history of metastatic chromophobe RCC is not well characterized and therefore, it may be difficult to quantify the therapeutic benefit associated with targeted therapeutic agents in this population. Several series investigate the survival of patients with metastatic chromophobe RCC and survival appears to vary widely with median survival ranging from 7 to 29 months. 10,11 The low incidence of metastatic chromophobe RCC, the limited insight into disease biology, and the fact that most targeted agents were mainly developed for and evaluated in patients with clear cell RCC are some factors that make characterization of the natural history and development of a standard therapeutic approach for this condition challenging. ${ }^{12}$

A better understanding of the biology of chromophobe RCC can enhance our ability to develop rational targeted strategies directed against specific pathways thought to contribute to this disease. Understanding the biochemical consequences of $V H L$ loss in hereditary and subsequently, sporadic forms of clear cell RCC was a critical step in the development of both VEGF- and mTOR-pathway antagonists. ${ }^{13}$ Similarly, emerging data from studies of a hereditary form of chromophobe RCC, the Birt-Hogg-Dube syndrome (BHD), may shed light on the biology of the sporadic form of the disease.

Table I: mTOR agents in metastatic chromophobe RCC: Summary of case reports.

\begin{tabular}{|c|c|c|c|c|c|c|c|}
\hline Case Report & Year & mTOR agent & Presentation & $\begin{array}{c}\text { Line of } \\
\text { Therapy }\end{array}$ & $\begin{array}{c}\text { Prior } \\
\text { Treatment }\end{array}$ & $\begin{array}{c}\text { Response by } \\
\text { RECIST }\end{array}$ & Outcome \\
\hline Present & $\mathbf{2 0 1 2}$ & Temsirolimus & $\begin{array}{c}\text { Recurrence } \\
\text { post- } \\
\text { Nephrectomy }\end{array}$ & 2nd & sunitinib & PR & $\begin{array}{c}\text { Alive } 5 \text { years with M1 disease, on } \\
\text { mTOR therapy for 4 years }\end{array}$ \\
\hline Paule & $\mathbf{2 0 1 1}$ & Temsirolimus & $\begin{array}{c}\text { Recurrence } \\
\text { post- } \\
\text { Nephrectomy }\end{array}$ & 3rd & $\begin{array}{c}\text { INF alpha, } \\
\text { sorafenib }\end{array}$ & NR & $\begin{array}{c}\text { Alive 3 years with M1 disease, on } \\
\text { mTOR therapy for 2 years }\end{array}$ \\
\hline Zardavas $^{10}$ & 2011 & Temsirolimus & $\begin{array}{c}\text { Recurrence } \\
\text { post- } \\
\text { Nephrectomy }\end{array}$ & 3rd & $\begin{array}{c}\text { sunitinib, } \\
\text { sorafenib }\end{array}$ & PR & $\begin{array}{c}\text { Alive 3 years with M1 disease, on } \\
\text { mTOR therapy for 1 year }\end{array}$ \\
\hline Larkin $^{1}$ & 2011 & Everolimus & $\begin{array}{c}\text { Recurrence } \\
\text { post- } \\
\text { Nephrectomy }\end{array}$ & 2nd & sunitinib & PR & $\begin{array}{c}\text { Alive } 5 \text { years with retroperitoneal } \\
\text { recurrence, remains on } \\
\text { everolimus for 2+ years }\end{array}$ \\
\hline
\end{tabular}

Abbreviations: NR- not reported, SD- syable disease, PR, partial response, M1- metastatic disease. 
A

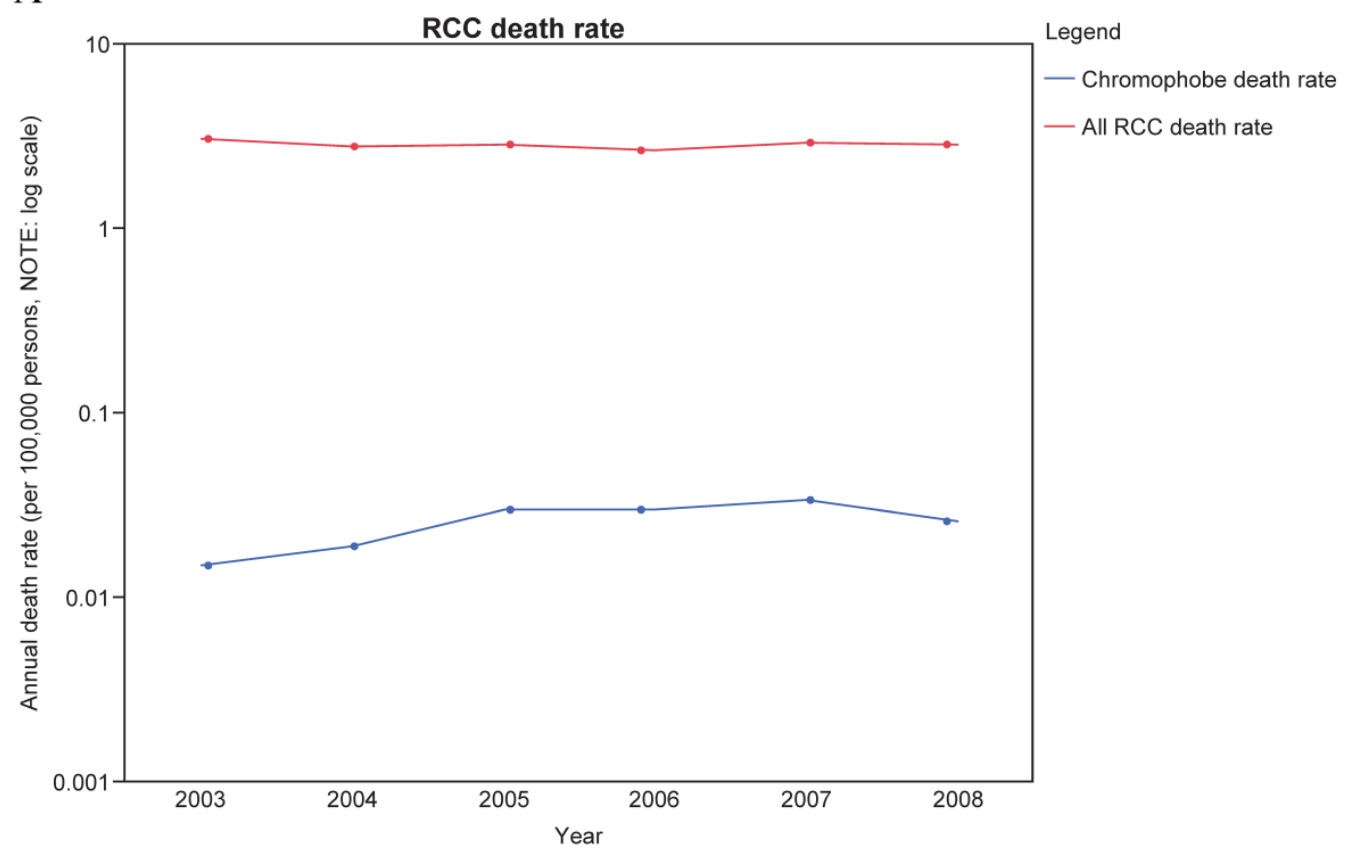

B

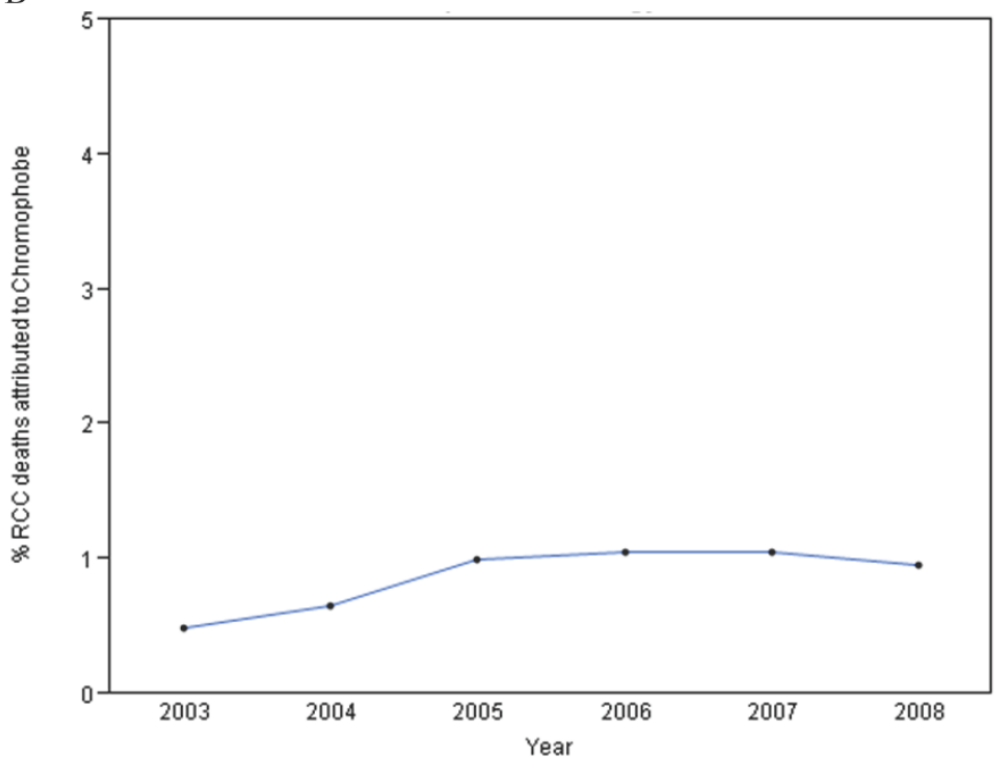

Figure 3. A: Annual death rate for RCC overall (red) and Chromophobe histology (Blue) from 2003-2008. Rate is per 100,000 persons and is age-adjusted to the US population in 2000. Note log scale. B: Percentage of RCC Deaths attributed to Chromophobe RCC histology from 2003-2008.

Patients with BHD are at risk for the development of bilateral, multifocal renal tumors and have germline inactivating mutations in the FLCN gene. Hasumi and colleagues from our institution demonstrated that folliculin loss leads to development of tumors with highly active $\mathrm{PI} 3 \mathrm{~K} / \mathrm{mTOR}$ pathways. ${ }^{1}$ Baba and colleagues have demonstrated that mTOR inhibition with rapamycin could reverse the pheno- type of polycystic kidneys and renal failure in a conditional FLCN knockout mouse model. ${ }^{14}$ Although the role of folliculin in non-hereditary forms of chromophobe RCC remains to be fully elucidated, these data provide a reasonable basis for the evaluation of the mTOR pathways and inhibitors of the PI3K/Akt/mTOR axis in chromophobe RCC. 
Moving forward, it may be nearly impossible to conduct a large randomized trial with metastatic chromophobe patients due to the rarity of this disease, as demonstrated by our SEER analyses. A reasonable approach might be to investigate agents whose use in chromophobe RCC is supported by preclinical studies, in carefully designed, well-coordinated multi-center phase 2 studies. Studies including patients with all types of non-clear cell histologies may also provide insight, as long as subtype stratification is performed. One such trial, ASPEN, a phase II trial of everolimus vs. sunitinib in metastatic, non-clear cell tumors, may provide some data on the activity of these agents in chromophobe RCC (NCI Trial ID: NCT01108445).

In conclusion, we support the evaluation of mTOR-directed therapy in chromophobe RCC with cautious enthusiasm; this approach is supported by both interesting anecdotal evidence and preclinical rationale. Due to the rarity of this disease, uncertainty about the biology and disease course, and lack of a coordinated approach to the management of this and other rare entities, there are many unanswered questions. We hope institutions across the globe will work together to determine the optimal systemic treatment for this challenging kidney cancer population.

\section{Acknowledgement}

Supported by the Intramural Research Program of the National Institutes of Health, National Cancer Institute, Center for Cancer Research. We would like to acknowledge Rabindra Gautam for his assistance with film review and administrative support. A special thanks goes to Dr. Sheldon Shuch and Maria Kwon for manuscript and figure editing.

\section{Abbreviations}

RCC: Renal Cell Carcinoma; RECIST: Response Evaluation Criteria In Solid Tumors; NCCN: National Comprehensive Cancer Network; VEGFR: Vascular Endothelial Growth Factor Receptor; VEGF: Vascular Endothelial Growth Factor.

\section{Competing Interests}

The authors have declared that no competing interest exists.

\section{References}

1. Hasumi Y, Baba M, Ajima R, et al: Homozygous loss of BHD causes early embryonic lethality and kidney tumor development with activation of mTORC1 and mTORC2. Proc Natl Acad Sci U S A 2009;106:18722-7.

2. Molina AM, Feldman DR, Ginsberg MS, et al: Phase II trial of sunitinib in patients with metastatic non-clear cell renal cell carcinoma. Invest New Drugs 2012 Feb;30(1):335-40.
3. Plimack E, Jonasch E, Bekele B, et al: Sunitinib in papillary renal cell carcinoma (pRCC): Results from a single-arm phase II study. J Clin Oncol 2010; 28:15s.

4. Choueiri TK, Plantade A, Elson P, et al: Efficacy of sunitinib and sorafenib in metastatic papillary and chromophobe renal cell carcinoma. J Clin Oncol, 2008; 26:127-31.

5. Stadler WM, Figlin RA, McDermott DF, et al: Safety and efficacy results of the advanced renal cell carcinoma sorafenib expanded access program in North America. Cancer 2010 Mar 1;116(5):1272-80

6. Hudes G, Carducci M, Tomczak $P$, et al: Temsirolimus, interferon alfa, or both for advanced renal-cell carcinoma. $\mathrm{N}$ Engl J Med, 2007; 356:2271-81.

7. Larkin JM, Fisher RA, Pickering LM, et al: Chromophobe renal cell carcinoma with prolonged response to sequential sunitinib and everolimus. J Clin Oncol 2011;29:e241-2.

8. Paule B, Brion N: Temsirolimus in metastatic chromophobe renal cell carcinoma after interferon and sorafenib therapy. Anticancer Res 2011;31:331-3.

9. Zardavas D, Meisel A, Samaras P, et al: Temsirolimus Is Highly Effective as Third-Line Treatment in Chromophobe Renal Cell Cancer. Case Reports in Oncology, 2011; 4:16-18.

10. Motzer RJ, Bacik J, Mariani T, et al: Treatment outcome and survival associated with metastatic renal cell carcinoma of non-clear-cell histology. J Clin Oncol, 2002; 20:2376-81.

11. Klatte T, Han KR, Said JW, et al: Pathobiology and prognosis of chromophobe renal cell carcinoma. Urol Oncol, 2008; 26:604-9.

12. Shuch B, Belldegrun A: Re: efficacy of sunitinib and sorafenib in metastatic papillary and chromophobe renal cell carcinoma. Eur Urol, 2008; 53:1085-6.

13. Yang JC, Haworth L, Sherry RM, et al: A randomized trial of bevacizumab, an anti-vascular endothelial growth factor antibody, for metastatic renal cancer. N Engl J Med, 2003; 349:427-34.

14. Baba M, Furihata M, Hong SB, et al: Kidney-targeted Birt-Hogg-Dube gene inactivation in a mouse model: Erk1/2 and Akt-mTOR activation, cell hyperproliferation, and polycystic kidneys. J Natl Cancer Inst, 2008; 100:140-54. 